\title{
Time to Stop Saying Geriatric Assessment Is Too Time Consuming
}

\author{
Marije E. Hamaker, Diakonessenhuis, Utrecht, the Netherlands \\ Tanya M. Wildes, Washington University School of Medicine, St Louis, MO \\ Siri Rostoft, Oslo University Hospital and University of Oslo, Oslo, Norway
}

Aging makes us increasingly unique. A group of older patients with cancer of identical chronologic age will demonstrate great heterogeneity with regard to vitality, comorbidity, functional status, physiologic reserves, and psychosocial functioning. ${ }^{1,2}$ Thus, age alone is an insufficient surrogate for biologic aging. Similarly, commonly used unidimensional measures, such as performance status or the American Society of Anesthesiologists classification, cannot fully do justice to this heterogeneity. ${ }^{3}$ Despite accumulating evidence regarding the value of geriatric assessment (GA) to encompass the diversity of older patients, it seems almost requisite in literature and presentations on GA in cancer care to state that it is too time consuming to implement in daily oncology practice. ${ }^{4-7}$ In our opinion, this complaint is ill founded and should be retired.

With the imminent aging of Western societies, the number of older patients with cancer is rapidly increasing. ${ }^{8}$ In fact, almost $70 \%$ of the patients sitting in front of you, as a cancer specialist, will be age 65 years or older by the year 2030. ${ }^{9}$ This demographic shift mandates a closer look at aging-related issues that older patients face, which persist and coexist with a new cancer diagnosis. In fact, such issues may be exacerbated by cancer symptoms and treatment-for example, increased risk of falls because of chemotherapy-related neuropathy in an older adult with limited mobility. ${ }^{10-12}$ As their patient population ages, cancer specialists increasingly have to make complex treatment decisions in older patients with comorbidities and functional impairment. This requires some familiarity and experience with geriatric care components. To address this demographic development, research on the incorporation of geriatric concepts into oncologic care began more than 20 years ago. ${ }^{13}$ Cancer specialists adopted the geriatric concept of frailty ${ }^{14}$-a state of decreased physiologic reserve caused by the accumulation of aging processes across multiple organ systems, which affects the patient's resistance to stressors such as cancer or cancer therapy. In addition, the GA was adopted for assessing the presence of impairments (eg, in cognition or mobility) and overall health status. Multiple resources now exist for oncologists wanting to implement a GA in clinical practice, such as the Web sites of the International Society of Geriatric Oncology, ${ }^{15}$ American Society of Clinical Oncology, ${ }^{16}$ Cancer and Aging Research Group, ${ }^{17}$ and Moffitt Cancer Center, ${ }^{18}$ as well as National Comprehensive Cancer Network older adult oncology guidelines. $^{19}$

In geriatric medicine, comprehensive GA (CGA) is "a multi-dimensional, interdisciplinary, diagnostic process to identify care needs, plan care, and improve outcomes of frail older people." $20($ p474) CGA encompasses multiple domains beyond a traditional medical assessment, including functional status, cognition, psychological health, and socioenvironmental factors. Tailored interventions are subsequently recommended, such as nutritional supplements or home nursing to help with medications. Furthermore, an important aspect of CGA is to discuss the patient's preferences and treatment goals so that the care plan reflects these crucial aspects of care. Although it is a time-consuming process, CGA has been proven to decrease mortality and care dependence, and is the essence of geriatric medicine. ${ }^{21}$ In geriatric oncology, a modified version of CGA, simply named GA, has been proposed and studied extensively in various tumor types and treatment settings. $^{22}$ The multidimensional character has been maintained, but with a simplified process, focusing primarily on identifying health issues that may affect treatment tolerance and prognosis.

In this form of GA, many of the data are collected by patient or caregiver self-report, sometimes electronically. Only certain components, including the cognitive screen and physical performance tests (eg, Timed Up and Go test ${ }^{23}$ ), require any health care provider time. Typically, such assessments can be performed by a nurse. Estimates of the total time required are 22 to 27 minutes, with 15 to 23 minutes being completed by the patient and caregiver and only 5 to 6 minutes by the health care provider. ${ }^{24-28}$ Provided that an intervention protocol is in place, there are no differences between assessments performed by a geriatrician or a trained health care worker in the proportion of patients for whom oncologic treatment decisions are altered, nor are there differences in the use of nononcologic interventions to optimize health status. ${ }^{29}$ Although completion of a series of screening instruments does not allow for actual clinical diagnosis of an underlying illness, such as depression or dementia, ${ }^{30}$ these instruments are quick and valid methods for identifying areas that may be impaired and acquiring an overall impression of a patient's health status.

What do the data show about the utility of GA in oncology? Even in patients with a good performance status, GA can identify multiple geriatric impairments. ${ }^{31,32}$ Additionally, among older patients beginning a course of chemotherapy, GA predicts toxicity. In the Cancer and Aging Research Group model, which includes GA parameters such as mobility and falls, the lowest-risk group had a $25 \%$ rate of grade 3 to 5 toxicity, whereas the highest-risk group had an $89 \%$ risk of grade 3 to 5 toxicity. The area under the 
curve of the model was $0.72 .^{33}$ In the Chemotherapy Risk Assessment for High Age Patients model, which also included several geriatric domains, separate models for grade $\geq 3$ hematologic and nonhematologic toxicities were developed, with $\mathrm{C}$ statistics of 0.76 and 0.66 , respectively. ${ }^{34}$

GA predicts 6-month mortality in older adults receiving chemotherapy, with at-risk or poor nutritional status and poor physical performance on the Timed Up and Go test each more than doubling the risk of early death (odds ratio, 2.77 and 2.55, respectively). ${ }^{35}$ GA is also predictive of postoperative morbidity in older adults with cancer undergoing surgery, where increasing age alone does not seem to be associated with complications in the elective setting. In a study of more than 400 patients with cancer, poorer functional status was associated with a $36 \%$ increased risk of complications after surgery, and being dependent in daily activities doubled the risk of prolonged hospitalization. ${ }^{36}$ In a cohort of older patients with colorectal cancer, frail patients had a 5-year survival of $24 \%$, compared with $66 \%$ for nonfrail patients, and being frail was a more important risk factor for death than stage III disease, whereas age was not associated with survival. ${ }^{37}$ In addition, several studies have shown that GA identifies previously unrecognized vulnerabilities that affect cancer specialists' decision making. In a systematic review of studies examining the impact of geriatric evaluation on treatment decisions, the median frequency with which the treatment plan was altered after GA was $39 \%$. $^{29}$ Most recently, the first study was published demonstrating improved outcome when treatment allocation was based on outcome of GA; in a randomized trial of patients with advanced non-smallcell lung cancer, GA-stratified treatment resulted in lower toxicity with similar survival. ${ }^{5}$ In the United States, a large ongoing community-based randomized trial will examine whether providing oncologists with their patients' GA data and relevant recommendations will affect treatment toxicity and quality of life (ClinicalTrials.gov identifier NCT02054741).

Traditional treatment paradigms focus on objectively measured factors such as tumor characteristics, computed tomography (CT) scans, biochemistry, and adherence to clinical guidelines that are on the basis of studies performed in cohorts of selected, often younger patients. ${ }^{38}$ If this focus becomes too dominant, we may forget to include patient preferences and perceptions in the decision-making process. Shared decision making can be facilitated by GA, because finding common ground in the patient's and physician's perceptions of the level of fitness and ability to tolerate treatment creates a good starting point for discussing treatment options. If an older patient is insistent on receiving therapy for cancer despite a high risk, GA may be particularly useful for the physician, because it provides a detailed explanation across all the domains of GA of why the risk is so high. In older patients, the best treatment option will depend just as much on subjective factors like the individual patient's preferences and priorities. Most treatment decisions involve tradeoffs (eg, accepting adverse effects of chemotherapy to increase the length of life). Studies have shown that functional and cognitive outcomes after treatment, such as the ability to live independently, may be more important than survival for older patients. ${ }^{39,40}$ Nevertheless, few studies in oncology have assessed these end points, focusing instead on what the physician finds most important: treatment toxicities, recurrence, and survival. However, when asking patient representatives about their top priorities for cancer research, the impact that cancer has on life, specifically psychological consequences and functioning, has been identified as the most important aspect. ${ }^{41}$ Another example that illustrates that physicians' and patients' perceptions deviate is that patient-reported toxicities are under-reported by physicians, even when collected within randomized trials. ${ }^{42}$ This is disturbing, because in such cases, the patient who is experiencing the toxicity has the correct answer.

Thus, we may need to take one step back and think again about what our job is: to offer the patient the best treatment on the basis of his or her preferences, values, and goals, as well as individualized risks and benefits, informed by GA. Taking a closer look at the patient is not merely time-consuming noise that interferes with an appropriate oncologic workup. This is especially true if the patient is older and has comorbidities, multiple medications, and cognitive impairment-factors that will influence every step of the treatment trajectory. Because the issues uncovered by GA can influence decision making by predicting survival as well as the likelihood of toxicity and other treatment-related complications, we believe it is unethical not to take the time to assess these aspects before treatment decisions are made. ${ }^{5,29,43-45}$ In addition to the harm caused to the individual patient, any cancer specialist will be aware of the time and resources required for dealing with treatment-related complications. In fact, the time required for GA, which may prevent complications by individualized toxicity prediction, is just a fraction of the cost of actual toxicity and complications.

Ultimately, time is money, including in clinical practice. The amount spent on staging and exploring disease characteristics is rapidly increasing. As summarized in Table 1, the relative cost of GA-expressed in terms of a nurse's salary for 1 hour-is small compared with many diagnostic procedures that are routinely used in oncologic workup. For example, a routine chest $\mathrm{x}$-ray costs 2.5

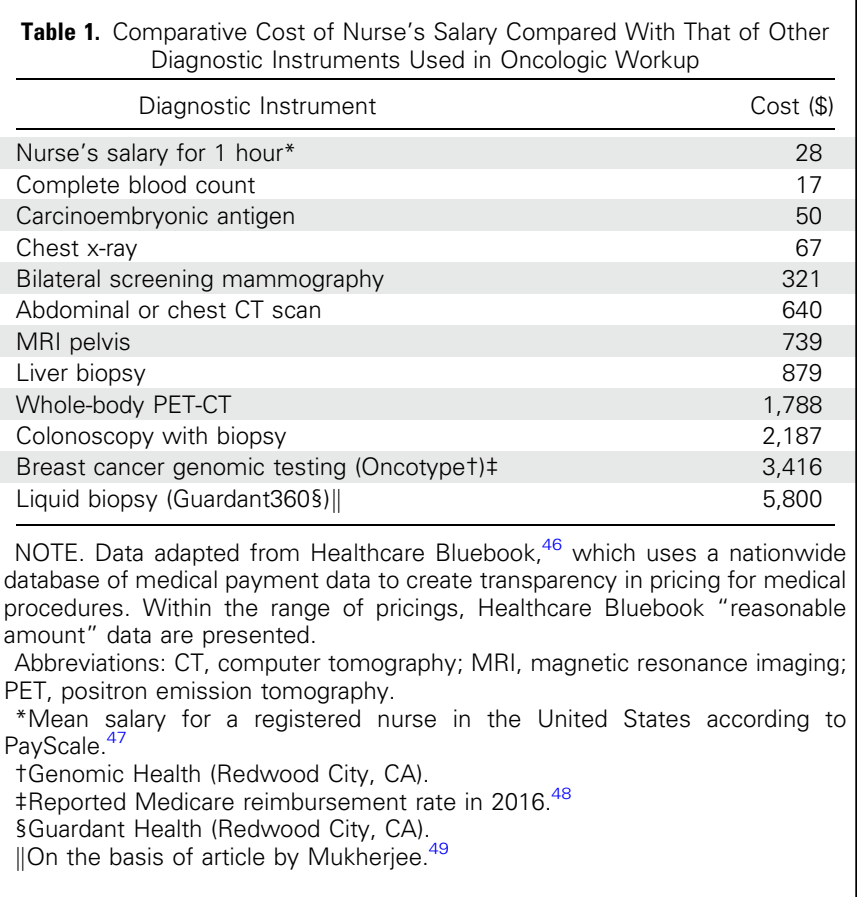


times as much; CT of the chest costs more than 20 times as much. Whole-body positron emission tomography-CT is nearly 65 times as expensive. Other assessments aimed at tailoring treatment, such as genomic testing, can cost more than 100 times more. Cost of health care is a complex issue. The time invested for GA in one cancer center may not necessarily result in cost savings for the hospital or cancer center director who makes the investment, because complications of cancer treatment are often managed in other hospitals, such as community hospitals. For this reason, we believe policymakers will play a key role in ensuring the routine implementation of GA in cancer care. Additionally, future studies in geriatric oncology should systematically assess the cost effectiveness of GA, both for the individual patient as well as on a societal level.

In conclusion, GA in the older patient with cancer can aid the physician in several ways: identifying impairments, clarifying patient priorities, predicting survival and toxicity risk, establishing a pretreatment baseline, and developing interventions. All these factors may influence treatment decisions for both the patient and medical team. Why is it deemed infeasible to take the extra time required for GA, when the cost of an additional imaging study or treatment or of dealing with toxicity is seldom the subject of debate? This results in care that is not tailored to the needs of older patients, potentially leading to unnecessary procedures or treatments or avoidable complications. Thus, it is in the interest of all stakeholders_patients, medical specialists, insurance companies, and policymakers - to demand an appropriate assessment of older patients with cancer.

\section{AUTHORS' DISCLOSURES OF POTENTIAL CONFLICTS OF INTEREST}

Disclosures provided by the authors are available with this article at jco.org.

\section{AUTHOR CONTRIBUTIONS}

Manuscript writing: All authors

Final approval of manuscript: All authors

\section{REFERENCES}

1. Jolly TA, Deal AM, Nyrop KA, et al: Geriatric assessment-identified deficits in older cancer patients with normal performance status. Oncologist 20:379-385, 2015

2. Caillet $P$, Laurent $M$, Bastuji-Garin $S$, et al: Optimal management of elderly cancer patients: Usefulness of the comprehensive geriatric assessment. Clin Interv Aging 9:1645-1660, 2014

3. Extermann $M$, Aapro $M$, Bernabei $R$, et al: Use of comprehensive geriatric assessment in older cancer patients: Recommendations from the task force on CGA of the International Society of Geriatric Oncology (SIOG). Crit Rev Onco Hematol 55:241-252, 2005

4. Palumbo A, Bringhen $S$, Mateos $M-V$, et al: Geriatric assessment predicts survival and toxicities in elderly myeloma patients: An International Myeloma Working Group report. Blood 125:2068-2074, 2015

5. Corre R, Greillier L, Le Caër H, et al: Use of a comprehensive geriatric assessment for the management of elderly patients with advanced non-small-cell lung cancer: The phase III randomized ESOGIA-GFPC-GECP 08-02 study. J Clin Oncol 34:1476-1483, 2016

6. Goede V, Bahlo J, Chataline V, et al: Evaluation of geriatric assessment in patients with chronic lymphocytic leukemia: Results of the CLL9 trial of the German CLL study group. Leuk Lymphoma 57:789-796, 2016

7. Kroep JR, van Werkhoven $E$, Polee $M$, et al: Randomised study of tegafururacil plus leucovorin versus capecitabine as first-line therapy in elderly patients with advanced colorectal cancer: TLC study. J Geriatr Oncol 6:307-315, 2015

8. Bluethmann SM, Mariotto $A B$, Rowland JH: Anticipating the "silver tsunami": Prevalence trajectories and comorbidity burden among older cancer survivors in the United States. Cancer Epidemiol Biomarkers Prev 25:1029-1036, 2016
9. Smith BD, Smith GL, Hurria A, et al: Future of cancer incidence in the United States: Burdens upon an aging, changing nation. J Clin Oncol 27:2758-2765, 2009

10. Ward $P R$, Wong $M D$, Moore $R$, et al: Fall-related injuries in elderly cancer patients treated with neurotoxic chemotherapy: A retrospective cohort study. J Geriatr Oncol 5:57-64, 2014

11. Tofthagen C, Overcash J, Kip K: Falls in persons with chemotherapyinduced peripheral neuropathy. Support Care Cancer 20:583-589, 2012

12. Gewandter JS, Fan $L$, Magnuson $A$, et al: Falls and functional impairments in cancer survivors with chemotherapy-induced peripheral neuropathy (CIPN): A University of Rochester CCOP study. Support Care Cancer 21:2059-2066, 2013

13. Yancik R, Ries LG: Cancer in the aged: An epidemiologic perspective on treatment issues. Cancer 68:2502-2510, 1991 (suppl)

14. Balducci L: Aging, frailty, and chemotherapy. Cancer Contr 14:7-12, 2007

15. International Society of Geriatric Oncology. http://siog.org

16. Cancer.net: For older adults. http://www.cancer.net/navigating-cancer-care/ older-adults

17. Cancer and Aging Research Group. http://www.mycarg.org/home

18. Moffitt Cancer Center: Senior adult oncology program.https://moffitt.org/ for-healthcare-providers/clinical-programs-and-services/senior-adult-oncologyprogram/

19. National Comprehensive Cancer Network: Senior adult oncology. https:// www.nccn.org/professionals/physician_gls/pdf/senior.pdf

20. Rubenstein LZ: Joseph T. Freeman award lecture: Comprehensive geriatric assessment-From miracle to reality. J Gerontol A Biol Sci Med Sci 59:473-477, 2004

21. Ellis G, Whitehead MA, O'Neill D, et al: Comprehensive geriatric assessment for older adults admitted to hospital. Cochrane Database Syst Rev 7: CD006211, 2011

22. Puts MTE, Santos B, Hardt J, et al: An update on a systematic review of the use of geriatric assessment for older adults in oncology. Ann Oncol 25:307-315, 2014

23. Podsiadlo D, Richardson S: The timed "Up \& Go": A test of basic functional mobility for frail elderly persons. J Am Geriatr Soc 39:142-148, 1991

24. Hurria A, Gupta S, Zauderer M, et al: Developing a cancer-specific geriatric assessment: A feasibility study. Cancer 104:1998-2005, 2005

25. Hurria A, Cirrincione CT, Muss HB, et al: Implementing a geriatric assessment in cooperative group clinical cancer trials: CALGB 360401. J Clin Oncol 29:1290-1296, 2011

26. McCleary NJ, Wigler D, Berry D, et al: Feasibility of computer-based selfadministered cancer-specific geriatric assessment in older patients with gastrointestinal malignancy. Oncologist 18:64-72, 2013

27. Williams GR, Deal AM, Jolly TA, et al: Feasibility of geriatric assessment in community oncology clinics. J Geriatr Oncol 5:245-251, 2014

28. Hurria A, Akiba C, Kim J, et al: Reliability, validity, and feasibility of a computer-based geriatric assessment for older adults with cancer. J Oncol Pract 12:e1025-e1034, 2016

29. Hamaker ME, Schiphorst AH, ten Bokkel Huinink D, et al: The effect of a geriatric evaluation on treatment decisions for older cancer patients: A systematic review. Acta Oncol 53:289-296, 2014

30. Mitchell AJ, Bird V, Rizzo M, et al: Diagnostic validity and added value of the Geriatric Depression Scale for depression in primary care: A meta-analysis of GDS30 and GDS15. J Affect Disord 125:10-17, 2010

31. Repetto L, Fratino L, Audisio RA, et al: Comprehensive geriatric assessment adds information to Eastern Cooperative Oncology Group performance status in elderly cancer patients: An Italian Group for Geriatric Oncology study. J Clin Oncol 20:494-502, 2002

32. Extermann M, Overcash J, Lyman GH, et al: Comorbidity and functional status are independent in older cancer patients. J Clin Oncol 16:1582-1587, 1998

33. Hurria A, Togawa K, Mohile SG, et al: Predicting chemotherapy toxicity in older adults with cancer: A prospective multicenter study. J Clin Oncol 29: 3457-3465, 2011

34. Extermann M, Boler I, Reich RR, et al: Predicting the risk of chemotherapy toxicity in older patients: The Chemotherapy Risk Assessment Scale for High-Age Patients (CRASH) score. Cancer 118:3377-3386, 2012

35. Soubeyran $P$, Fonck $M$, Blanc-Bisson $C$, et al: Predictors of early death risk in older patients treated with first-line chemotherapy for cancer. J Clin Oncol 30: 1829-1834, 2012

36. Audisio RA, Pope D, Ramesh HS, et al: Shall we operate? Preoperative assessment in elderly cancer patients (PACE) can help-A SIOG surgical task force prospective study. Crit Rev Oncol Hematol 65:156-163, 2008

37. Ommundsen $N$, Wyller TB, Nesbakken A, et al: Frailty is an independent predictor of survival in older patients with colorectal cancer. Oncologist 19: 1268-1275, 2014 
38. Scher KS, Hurria A: Under-representation of older adults in cancer registration trials: Known problem, little progress. J Clin Oncol 30:2036-2038, 2012

39. Fried TR, Bradley EH, Towle VR, et al: Understanding the treatment preferences of seriously ill patients. N Engl J Med 346:1061-1066, 2002

40. Akishita M, Ishii S, Kojima T, et al: Priorities of health care outcomes for the elderly. J Am Med Dir Assoc 14:479-484, 2013

41. Corner J, Wright $D$, Hopkinson J, et al: The research priorities of patients attending UK cancer treatment centres: Findings from a modified nominal group study. Br J Cancer 96:875-881, 2007

42. Di Maio M, Gallo C, Leighl NB, et al: Symptomatic toxicities experienced during anticancer treatment: Agreement between patient and physician reporting in three randomized trials. J Clin Oncol 33:910-915, 2015

43. Wildiers $H$, Heeren $P$, Puts $M$, et al: International Society of Geriatric Oncology consensus on geriatric assessment in older patients with cancer. J Clin Oncol 32:2595-2603, 2014
44. Hurria A, Mohile $S$, Gajra A, et al: Validation of a prediction tool for chemotherapy toxicity in older adults with cancer. J Clin Oncol 34:2366-2371, 2016

45. Lichtman SM: Polypharmacy: Geriatric oncology evaluation should become mainstream. J Clin Oncol 33:1422-1423, 2015

46. Healthcare Bluebook. https://healthcarebluebook.com

47. PayScale. http://www.payscale.com/

48. GenomeWeb: CMS restores reimbursement rate for Oncotype DX breast cancer test. https://www.genomeweb.com/molecular-diagnostics/cms-restoresreimbursement-rate-oncotype-dx-breast-cancer-test

49. Mukherjee S: The cancer biopsy of the future could be a simple blood test. http://fortune.com/2016/06/05/asco-guardant-liquid-biopsy/

DOI: https://doi.org/10.1200/JCO.2017.72.8170; published at jco.org on June 19, 2017. 


\section{AUTHORS' DISCLOSURES OF POTENTIAL CONFLICTS OF INTEREST}

Time to Stop Saying Geriatric Assessment Is Too Time Consuming

The following represents disclosure information provided by authors of this manuscript. All relationships are considered compensated. Relationships are self-held unless noted. I = Immediate Family Member, Inst = My Institution. Relationships may not relate to the subject matter of this manuscript. For more information about ASCO's conflict of interest policy, please refer to www.asco.org/rwc or ascopubs.org/jco/site/ifc.

Marije E. Hamaker

Consulting or Advisory Role: Roche

Tanya M. Wildes

Honoraria: Carevive Systems

\section{Siri Rostoft}

No relationship to disclose 
Hamaker, Wildes, and Rostoft

\section{Acknowledgment}

All authors contributed equally to this work. 